\title{
De la linéarité énumérative à la configuration descriptive : le discours littéraire des écrivains roumains d'expression française
}

\author{
Cecilia CONDEI \\ Université de Craiova \\ Roumanie
}

\begin{abstract}
Résumé. La présente étude repose sur la problématique de la description, type de texte dont l'hétérogénéité est unanimement reconnue, pour observer le fonctionnement de l'énumération sous ses formes de base (liste et inventaire) en rapport avec la description proprement dite. Deux perspectives nous guident : discursive et textuelle, leur nécessité est imposée par la considération du discours et du texte comme inséparables et par la constitution du corpus d'étude : le discours littéraire romanesque des écrivains roumains d'expression française. Notre parcours illustre et confirme deux critères opératoires dans la classification des formes de l'énumération et insiste sur les aspects discursifs liés aux expressions du point de vue et aux changements discursifs exigés par les séquences images photographiques issues de notre corpus.
\end{abstract}

Abstract. The present study bases on the problem of the description, the type of text whose heterogeneousness is unanimously recognized, to observe the functioning of the enumeration under its base forms (list and inventory) in touch with the description cleanly said. Two perspectives guide us: discursive and textual, their necessity is imposed by the consideration of the speech and the text as inseparable and by the constitution of the corpus of study: the romantic literary speech of the Rumanian writers of French expression. Our way confirms two operating criteria in the classification of the forms of the enumeration and insists on the discursive aspects associated to the expressions of the point of view and to the discursive changes required by sequences - photographic images.

Mots-clés : Discours littéraire, linéarité, description, énumération, configuration

Keywords : Speech literary, linearity, description, enumeration, configuration

\section{Cadrage théorique}

Envisager la problématique du descriptif et des opérations qui le soutiennent signifie toucher l'énumération. Quant à la description, elle «ne relève pas d'un genre, c'est un type de discours [...] est description la déclinaison d'un paradigme latent (ayant déjà un principe organisateur), qui opère dans une liste, une énumération, d'une manière plus générale dans une expansion ramenée ensuite à l'unité par la présence du "pantonyme » qui nomme l'objet ou la catégorie d'objets dont les aspects nombreux ont été explorés » (Hamon 1990, 2)

Le propos de Philippe Hamon, avec la définition de la description, représente le cadre de notre réflexion sur les formes descriptives présentes dans le discours littéraire romanesque. Le décryptage de l'énumération suppose la prise en compte des éléments textuels qui la signalent et la 
régissent, mais l'observation des sources énonciatives qui y sont concernées permet de dégager des éléments de fonctionnement qui dépassent le plan du texte et s'inscrivent dans la discursivité. L'analyse des œuvres des écrivains roumains d'expression française (Oana Orlea, Maria Mailat, Matei Visniec, Rodica Iulian, Dumitru Tsepeneag, Liliana Lazar) privilégie deux positions : l'une, discursive, pour distinguer l'expression du point de vue qui découle d'une procédure descriptive, et l'autre textuelle, pour observer les opérations de base qui assurent la lisibilité d'une séquence descriptive, non sans souligner les problèmes de classification de l'énumération et les principaux critères qui facilitent cette classification.

La liste et l'inventaire, en tant qu'éléments énumératifs sont les formes basiques de la description. Les dictionnaires (entre autres, Trésor de la Langue française, version informatisée) expliquent leurs sens insistant, pour l'inventaire, sur sa rigueur ( B. - [À propos d'éléments, matériels ou non, qui présentent une similitude de nature ou de fonction] Dénombrement d'éléments, groupés par catégories, qui constituent un ensemble cohérent, à un moment donné; $p$. méton., ensemble des éléments ainsi dénombrés ou répertoire des éléments dénombrés ») et pour la liste, sur sa mise en page ( A. - 1. Suite continue, hiérarchisée ou non, de noms (de personnes ou d'objets) ou de signes généralement présentés en colonne. »).

À un bref regard, la liste et l'inventaire, de par leur simplicité, ne concernent pas trop le côté ornemental du texte littéraire. Anne -Marie Paveau et Laurence Rosier affirment la marginalité de la liste et insistent à la considérer comme un "modèle primitif d'écriture », " en marge de la syntaxe », «dans les marges de la production textuelle» (2009, 114-115), non sans attirer l'attention sur quelques situations où la liste et l'inventaire, associés à l'accumulation, intéressent les littéraires.

Pour notre part, ayant en vue l'analyse du discours littéraire, nous abordons les différentes formes d'énumération qui se trouvent à la base de la description, suivant notre perspective de linguiste qui, sans abandonner son objectif discursif, visite le territoire littéraire. Si pour le domaine du droit ou pour l'administration, il est peut-être possible de différentier la liste de l'inventaire, sur la base de la rigueur et de l'organisation par catégories du deuxième, le discours littéraire pose d'emblée la difficulté de différenciation, tout en laissant quelques portes ouvertes pour des tentatives de ce genre.

\section{Problèmes de classification de l'énumération}

Parler d'une classification impose la prise en compte de l'organisation des éléments concernés et, ce qui est une spécificité dans notre cas, la délimitation de ces éléments. 


\section{Critère organisationnel}

Un premier obstacle, signalé par Jean-Michel Adam et André Petitjean, tient de la mise en séquence qui distingue la liste «énumération pure ", aléatoire, de la description, capable de supporter des "procédures de mise en séquence» $(1989$, 61). À partir de cette distinction, nous considérons que le degré d'organisation peut devenir critère de distinction, ce qui permettrait de traiter la liste en tant que forme de l'aléatoire, l'inventaire comme forme avec une organisation faiblement manifestée et la description proprement dite comme une séquence bien bâtie à l'aide des organisateurs. Les situations sont illustrées dans 1), 2),3).

(1)[Contexte discursif : Devant la télé qui transmet, à l'époque communiste roumaine, un message révolutionnaire du président de la République, Kouty, un adolescent, Marc, sa mère, Léontine, ancienne enseignante devenue femme de ménage et une vieille dame, Marie-Louise, une des ces clientes, regardent amusés l'écran, après avoir coupé le son.]

Marie-Louise ouvrit un tiroir et en sortit une grosse enveloppe pleine d'autocollants, qu'elle donna à Marc :

- Colle-moi ça sur l'écran, mon grand.

Avec à peine une hésitation, Marc s'agenouilla devant l'écran. Deux grosses coccinelles recouvrirent les yeux de Kouty. Sa bouche, à lèvre inférieure charnue, légèrement tordue, continua à s'agiter sous les deux coccinelles. Léontine eut la nausée.

- Dépêche-toi, dit Marie-Louise.

Marc colla sur la bouche de Kouty un très beau poisson aux nageoires roses. De deux côtés du poisson, les mâchoires continuaient de bouger. Des fleures, des Indiens, des champignons, un serpent, une voiture de course, une botte de carottes, deux petits soleils, deux oiseaux mirent fin au visage de Kouty. (Orlea 1986, 75)

(2)[Contexte discursif: Une enseignante ressemblant fortement à la Première Dame de l'époque communiste roumaine va devenir son sosie. Les services chargés au recrutement préparent longuement cette embauche agissant de manière à ce que l'enseignante Léontine, plusieurs fois licenciée, devienne femme de ménage dans les maisons des policiers de la zone résidentielle pour qu'elle puisse ainsi mieux apprécier la position de sosie. Avant d'être embauchée dans une telle famille, on lui explique le rôle.]

Les maisons de ce genre sont généralement réservées aux femmes de ménage expérimentées, alors, n'oubliez surtout pas que vos connaissances d'intello n'intéressent personne. Frotter, laver, aspirer, faire briller, repasser, silence et docilité, c'est tout ce que l'on vous demande. (Orlea 1986, 44) 
(3) Une bonne demi-heure de marche dans les bois est nécessaire pour arriver jusqu'au lac. Il faut d'abord longer les collines qui surplombent Slobozia, et s'enfoncer plus profondément dans les taillis de hêtres et de chênes. À son approche, le sentier se fait sinueux, la chênaie devient plus dense. Puis quand le marcheur, convaincu de s'être égaré, songe à rebrousser chemin, soudain, au détour d'un bosquet, il l'aperçoit enfin : le lac. Un ruisseau qui serpente à travers les collines vient s'y jeter. Gonflé à la fin de l'hiver par la fonte des neiges, il n'est à la belle saison qu'un mince filet d'eau. (Lazar 2009, 11)

Dans (1) c'est l'aléatoire qui domine, fait souligné par «l'enveloppe pleine d'autocollants » que le garçon commence à placer sur l'écran. Aucun lien catégoriel entre "poisson aux nageoires roses», «Indiens», «champignons». Comme l'activité impose la vitesse, car l'image du Conducator peut disparaittre, aucune intention non plus de choisir un ordre quelconque au moment où l'on extrait les autocollants.

L'argument pour encadrer (2) comme inventaire tient du fait que l'énumération des activités à entreprendre se présente sous une forme finie, marquée explicitement par «tout» et aussi du fait que le contenu est divisé en deux groupes. Le premier, "frotter, laver, aspirer, faire briller » est centré sur l'activité à déployer, sur le faire, le deuxième, «silence et docilité » sur l'attitude que l'on doit avoir. L'inventaire est cette fois-ci une accumulation d'éléments rangés selon des critères précis et non pas d'une manière aléatoire.

Pour ce qui se présente sous (3), la séquence est une description. La représentation qu'elle propose se construit lors du déplacement « dans le bois », déplacement ancré temporellement "une bonne demi-heure de marche", selon un certain parcours imposé : "longer les collines", «s'enfoncer dans les taillis », suivre un sentier «sinueux ». Description du chemin, mais aussi du "marcheur», de son comportement, de ses sentiments. La focalisation jusqu'ici bornée sur deux éléments: le promeneur et le chemin, se fixe ensuite sur le statique - le lac, devenu thème-titre.. Ce qui suit s'organise autour de ce noyau «le corps de la description est l'expansion (plus ou moins développée) du thème-titre » (Adam, Petitjean 1989, 106).

Si dans 1) c'est l'aléatoire qui domine, dans (2) la structuration, encore faiblement manifestée permet pourtant de parler d'un plan, pendant que les organisateurs de (3), «d'abord» et« puis» opèrent une délinéarisation du discours. D'ailleurs, sorte de "degré zéro de la procédure descriptive» (Adam 2011, 68), l'énumération a pour effet immédiat une linéarisation nuisible pour la lecture interprétation et, en somme, pour la construction du sens. Les organisateurs rétablissent l'équilibre.

Jean-Michel Adam parle de "plans de texte qui organisent l'information en fonction de listes à saturation prévisible (quatre points 
cardinaux, quatre saisons, cinq sens, ordre alphabétique ou numérique). Ces listes, tout en traduisant un procédé de sériation servant de balisage à la production comme à la lecture font appel à des principes d'organisation différents: de type alphabétique ou numérique, topographique et/ou chronologique » $(1989,66)$.

\section{La délimitation comme critère de typologisation}

Un second obstacle s'oppose à la mise en forme de l'énumération : la difficulté de déterminer ses frontières.

Ainsi, prenant comme critère l'achèvement/l'inachèvement, AnneMarie Paveau et Laurence Rosier considèrent que «la liste est systématique, mais jamais exhaustive. Mais elle est aussi clôturée de façon arbitraire: une liste de morts sur un monument semble achevée, tout comme la liste des victimes d'un crash aérien ou d'un attentat; mais une liste de course est-elle fermée? Un inventaire, en revanche, sera défini comme un listage exhaustif et clos. » $(2009,114)$

Si dans un autre type de discours que celui littéraire, le critère fonctionne sans aucun obstacle, puisque l'inventaire, de par son rôle, est censé tout indiquer, et même sans oublier d'expliciter la quantité d'objets recensés, comme on le fait pour l'inventaire des biens exigé par la procédure successorale, le texte littéraire, ayant d'autres visés, utilise différemment les formes énumératives. Dans ce qui suit, la liste aurait été facilement identifiée selon le critère achèvement/inachèvement:

(4) - Ce sont des dresseurs !

- Des dresseurs de quoi ? [...]

Je reconnais ne pas comprendre. Le jeune homme blond assis à mes côtes qui m'avait été présenté comme étant 'gentil, mais un peu autiste sur les bords', sort de son mutisme :

- Les chevaux, les singes, les chiens, les chats, les lions, les panthères, les tigres, les éléphants, les rats, les perroquets, les colombes, les crocodiles...- Il reprend sa respiration : - Enfin, toutes les créatures de Dieu, sauf l'homme. (Orlea 2007, 18-19)

L'énumération des animaux dressés s'arrête, des points de suspension marquent l'inachèvement (cf. Anne-Marie Paveau et Laurence Rosier 2009, 115) et pourtant la liste est close, car il est impossible et inutile de mentionner tous les noms concernés, mais on résume explicitement : «Enfin, toutes les créatures de Dieu, sauf l’homme ».

La liste de l'exemple (1), même bien clôturée, ne peut pourtant pas être classée comme inventaire. Si la rigueur concernant la mention de la quantité apparaît dans quelques situations : deux grosses coccinelles, un serpent, une voiture de course, une botte de carottes, deux petits soleils, 
deux oiseaux, pour les fleurs les Indiens et les champignons, on reste bien dans l'approximation qui expulse le morceau de la catégorie « inventaire »

Donc, nous avons déjà souligné, ce que les dictionnaires retiennent comme démarcatif entre liste et inventaire, c'est la rigueur du dernier, le fait qu'il peut entrer dans des constructions des langues de spécialité, le vocabulaire juridique, par exemple. Cela laisse inférer que l'exactitude de l'inventaire est une marque. Dans ce qui suit, une séquence qui pouvait bien être classifiée comme inventaire, ne l'est pourtant pas, à cause des approximations, d'ailleurs explicites :

(5) J'ai dix serpents, trois tortues, une vingtaine de souris blanches, quelques grenouilles (elles sont si perfides que je n'arrive jamais à savoir combien elles sont), deux escargots, un chien, trois chats (un chat noir, un chat blanc et un chat roux), deux coqs de Cochinchine, un perroquet, deux tourterelles, une famille de scorpions, une famille de lapins, une soixantaine de petits poissons rouges dans un grand aquarium, un hérisson, au moins une centaine de coccinelles et trente papillons diurnes, merveilleusement colorés. (Visniec 1996, 30)

«Quelques », « une soixantaine », « au moins » sortent l'énumération de la catégorie inventaire. En plus, l'inventaire suppose la catégorisation interne qui n'existe pas ici.

Le type suivant d'énumération peut être rangé comme inventaire, même si les informations sur la quantité sont absentes:

(6) Quel genre de flic ? Lorsqu'elle referma le tiroir, les couverts bien rangés dedans: cuillers, fourchettes, couteaux, petites cuillers, petites fourchettes, petits couteaux, elle avait cessé de se poser la question.

Je range les cuillers, les fourchettes, les couteaux, les petites cuillers, petites fourchettes, petits couteaux dans un tiroir que je viens de nettoyer, c'est le tiroir d'un meuble bas, en chêne, qui fait partie de l'ameublement de cette cuisine où je fais le ménage comme employée de maison. (Orlea 1986, 46)

L'absence d'article dans le premier paragraphe et la présence de l'article défini, dans le deuxième, deviennent des symboles du connu et opèrent des démarcations précises entre les catégories d’objets. En plus, la représentation que cette liste déclenche est celle d'un groupe limité à ces trois types d'objets, indispensables pour manger : cuillers, petites cuillers, fourchettes, petites fourchettes, couteaux, petits couteaux. C'est donc sur une représentation collective que se construit la liste. En quelque sorte la suivante se construit de la même manière, sauf qu'elle c'est difficile la clôturer, car tout «jardin zoologique» possède un certain nombre d'animaux, aucun critère de le limiter. La présence d'un évaluatif peut aider à la construction d'une limite, puisqu'une appréciation du type «beau jardin » a une valeur positive qui conduit à la conclusion que la quantité et la variété d'objets peuvent assurer une liste finie. 
(7) Qu'est-ce qu'on a ramassé jusqu'à présent, deux lapins, quatre souris, un serpent, un lézard, un papillon, une grenouille, une tortue, un hérisson, deux escargots et un écureuil, cela fait un beau jardin zoologique! (Visniec 1996, 104)

Même si l'on ne peut pas prévoir la fin d'une énumération, un «et», « employé isolément, peut marquer la clôture » (Adam 2011, 70).

Nous ajoutons plusieurs situations indiquant le marquage du début ou de la fin de l'énumération.

a) D'abord quelques types de syntagmes, centrés sur des mots indiquant la fin, partout, tout (forme adverbiale ou nominale), entier/ère, encore, etc. Un « tout », placé avant, comme dans 8a) ou après, comme dans 9d) et 9e), l'énumération indique sa clôture :

(8)a) Il n'y a pas d'abri contre la pluie-animal, les gens ont tout essayé : les parapluies métalliques, les pèlerins cuirassés, les refuges souterrains blindés, la révolte et le silence. (Visniec 1996, 52)

Ou encore :

b) Les papillons carnivores ont été chassés de la ville par les escargots pestilentiels. Ils sont sortis de partout: des entrailles de la terre, des canaux, des caves, des égouts. (Visniec 1996, 49)

c) J'ai maintenant presque mille poèmes sur les oiseaux, les arbres, les rochers, le vent, la neige, la lune, les étoiles, les nuages, les arcs-en-ciel, l'herbe ou la prairie, ou mille autres choses encore. (Visniec 1996, 19)

d) Regardez-vous, comme vous aimez acheter de la viande! Du porc, du veau, du poisson, des escargots, des volailles, tout est bon pour votre corps de chair. (Visniec 1996, 81)

e) J'ai si peur de la douleur. Pourtant: plonger la main dans l'huile brûlante, casser une vitre avec le poing, éteindre une cigarette sur mon avant-bras, tout cela je l'ai fait pour m'endurcir. (Orlea 2007, 70)

C'est encore le cas de "toutes les créatures du Dieu », (4), où la limite est soulignée par un organisateur: «enfin ». Pour ce qui est du début, les deux points sont de loin le procédé le plus utilisé.

b) marques de ponctuation ou typographiques: point, deux-points, parenthèses, italiques, tirets.

(9) a) Quant à la musique, il est grand amateur de Vivaldi, Corelli, Händel. Il digère difficilement Debussy. (Visniec 1996, 97)

b) Le souvenir de la ville détruite, à peine troublé par le temps, par les années écoulées, persiste en elle avec tant de force qu'elle en subit le fardeau sans jamais pouvoir se dérober : la Maison des Tilleuls, la Maison du Puits, celle des Deux Chats, la Maison des Iris, de la Louve, le Prieuré, l'Église et ses fresques bleues du quinzième siècle, la Maison des Jumeaux 
avec son vieux porche, la Maison jaune, la blanche, la rose... (Orlea 2007, 32)

c) Les fonctions vitales du cerveau (la mémoire, l'imagination, le raisonnement) ne sont en aucune manière affectées. (Visniec 1996, 72)

d) Toute atteinte (dommage matériel, pillage, incendie) à un centre de lavage sera sanctionnée par un lavage total de cerveau du ou des coupables. (Visniec 1996, 78)

e) Le lavage de cerveau nous offre la porte d'un abri sûr contre les cauchemars de toutes sortes, contre la folie, contre la double personnalité. (Visniec 1996, 72)

L'indication de clôture peut être également réalisée par le syntagme « dét.+choses », comme dans $8 \mathrm{c}$ ). À part les tirets, les autres marques sont bien présentes dans notre corpus.

Un rapport peut être établi entre le choix du marquage et les informations quantitatives contenues dans la liste. Les segments entre parenthèses sont plus courts, la liste est maigre. Si l'on la compare avec celle qui suit les deux points. La liste parenthèse est sentie comme un ajout explicatif "dommage matériel, pillage, incendie», ajout porteur des informations nouvelles dont on ne peut généralement pas s'en passer, ou comme un ajout restrictif.

\section{Perspective discursive}

Un premier aspect à prendre en compte est l'expression du point de vue qui découle d'une procédure descriptive. Dans ce qui suit, le point de vue change tout en marquant son changement par répétition de certains éléments :

(10) Quel genre de flic ? Lorsqu'elle referma le tiroir, les couverts bien rangés dedans: cuillers, fourchettes, couteaux, petites cuillers, petites fourchettes, petits couteaux, elle avait cessé de se poser la question.

Je range les cuillers, les fourchettes, les couteaux, les petites cuillers, petites fourchettes, petits couteaux dans un tiroir que je viens de nettoyer, c'est le tiroir d'un meuble bas, en chêne, qui fait partie de l'ameublement de cette cuisine où je fais le ménage comme employée de maison. (Orlea 1986, 46)

La modification de la personne, le passage de la troisième à la première personne, ainsi que la présence des déterminants dans le deuxième paragraphe complètent linguistiquement l'expression du point de vue.

Un deuxième aspect est généré par ce que Philipe Hamon appelle «boîte photographique ». Il conçoit le texte descriptif comme «structuré par le système de la boite photographique. Au début, la chambre [...] puis l'espace intermédiaire, le diaphragme, lui-même architecturalement structuré : la fenêtre » (Hamon 1990, 3). Cette remarque, prenant comme 
point de départ un poème de Victor Hugo, La Pente de la rêverie, pose des problèmes d'interprétation: l'idée d'une construction à caractère photographique, peu ou moins distinguable dans toute description, et, ensuite, la direction spatio-temporelle mise en jeu.

Pour illustrer le caractère photographique, le discours littéraire que nous visitons, se montre bien généreux :

(11) [Cadrage discursif: dans une gare, on tourne un film. Les personnages sont en possession du synopsis et chacun essaie d'entrer dans son rôle, comme le fait ce chef de gare]

Un homme dort.

Il garde les yeux fermés, mais son visage, en gros plan, est plutôt celui de quelqu'un qui attend, qui guette quelque chose. Peut-être qu'il ne dort pas. Qu'il fait semblant.

On découvre le lit, le corps étendu comme sur une civière. Pour ne pas dire un cercueil. Les mains jointes sur la poitrine, sur la couverture garance. Au long du lit, un mur nu.

À présent, l'homme a les yeux ouverts, l'impression d'attente est encore plus forte. On suit son regard qui se déplace de droite à gauche.

Sur la table de nuit, de menus objets : un bouton, un stylo, un sifflet, un réveille-matin, une gomme, une montre-bracelet, une photo sur laquelle on aperçoit une femme, assez jeune. On la reverra en chair et en os.[...] Je dors.

En gros plan, mon visage est plutôt celui de quelqu'un qui attend, qui guette quelque chose. D'accord. Peut-être que je ne dors pas. Que je fais semblant. Alors je rouvre les yeux ou je bats seulement les cils ?Je fais tremblant mes cils. Imperceptiblement. La caméra est braquée sur ma gueule. C'est après qu'on voit le lit et, naturellement, mon corps étendu comme sur une civière. Il ne veut pas dire cercueil. Ça le regarde, passons! Les mains jointes sur la poitrine, la couverture rouge, etc.

Mes yeux sont ouverts. À présent, l'impression d'attente est encore plus forte. Mon regard se déplace : de droite à gauche. Celui du lecteur: de gauche à droite !...

Je regarde les menus objets qui se trouvent sur la table de nuit: un bouton, un sifflet, un réveille-matin, une photo. De Marie-Christine, naturellement. (Tsepeneag 1985, 9-13)

Le même contenu imaginaire vu, premièrement d'une manière neutre, de l'extérieur, en utilisant la troisième personne apparaît, en deuxième position, de la perspective de celui qui décrit ses actions. L'objectif photographique regarde l'homme, ensuite c'est l'homme qui se regarde à travers cet objectif.

La liste de menu objets du premier paragraphe a un rôle différent de celle du deuxième paragraphe, même si son contenu est presque le même. D’une liste informative, une énumération d'objet que la caméra doit montrer, selon la vision du metteur en scène, elle devient une liste 
suggérée, plus maigre comme contenu parce que ce n'est pas l'entier contenu qui compte, mais seulement quelques détails, importants pour la compréhension ultérieure.

Même technique de la caméra qui bouge dans un décor ou braque sur quelqu'un, dans le roman de Maria Mailat. Se voir d'un dehors de soi devient cette fois-ci déclencheur de récit pour le personnage narrateur, Mina Bailar :

12) Par un heureux concours de circonstances, je découvre le Foyer pour immigrés israélites. Assise dans la salle d'attente, j’observais un couple de Falasha: elle, excessivement maigre, visage marqué par une pénible maturité sans âge, belle, lui, l'éternel gamin. De temps en temps, il se mettait à se balancer d'avant en arrière. Sa femme lui posait la main sur l'épaule pour le calmer.

En face d'eux, une Polonaise robuste gardait sur ses genoux sa fille aux blondes nattes, occupée à bercer sa Barbie. Dans un coin, installée sur le rebord d'une chaise, Mina Bailar : vêtements gris, mal coupés, sans grâce. Mon pull avait été tricoté par ma mère. Fil d'angora recyclé depuis trois générations. Mon sac à dos protégeait un roman écrit en roumain et tapé à la machine. On aurait dit que le papier pelure avait été arraché à ma peau. Ces feuilles occupaient peu de place. (Mailat 2003, 83)

En ce qui concerne la direction spatio-temporelle, nos exemples ont plusieurs fois relevé ce regard sur soi, une direction de l'extérieur vers le descripteur qui s'auto-décrit traduisant en page-espace sa temporalité discursive.

\section{Perspective textuelle}

La description a été conçue "comme un mouvement de dérivation ou d'expansion lexico-sémantique à partir d'un terme-noyau » (Jeandillou 1997, 149). Jean-Michel Adam et André Petijean, pour leur part, précisent

que «la reconnaissance et l'établissement d'un noyau descriptif hiérarchique joue un rôle essentiel dans le traitement cognitif du texte ou d'une simple séquence descriptive. » $(1989,110)$. Pour ce qui de l'opération descriptive, les linguistes reprennent la définition de M.-J. Borel: «le discours indique ce dont il va parler au moyen d'un signe indicateur qui renvoie à un ensemble de significations préconstruites » (1989, 113-114) insistant sur le processus qui déclenche la classe-objet et qui se trouve à la base de l'ancrage. C'est le rôle de «viande » dans 8d), de «souvenir des maisons » dans 9b), ou « fonctions du cerveau », dans 9c).

Les opérations de base qui assurent la lisibilité d'une séquence descriptive sont l'ancrage, l'affectation et la reformulation.

L'ancrage représente donc «le choix d'un thème-objet du discours » (1989, 105). Cela va dans la direction de Philipe Hamon et de l'explication du mécanisme du résumé, que celui-ci propose. 
(13) Coucher du soleil. Dans les cheminées, à l'ombre des surplombs, dans les recoins peu visités par le soleil, des cascades rouge foncé contrastent avec le rouge plus pâle qui couvre la paroi lisse, avec la couche de glace bleuâtre sous laquelle dort l'eau du lac. (Iulian 2001, 9596)

Le premier syntagme est un élément essentiel pour l'activation des savoirs déjà existants, mémorisés, sorte de "préconstruits culturels » (Adam, Petitjean 1989, 114). Le mécanisme d'activation de l'opération d'ancrage se manifeste à plusieurs niveaux. À part le thème-titre, peut apparaître le titre-genre textuel, comme plus bas, où la disposition du texte en colonnes assure un supplément important pour le classifier comme « liste ».

(14) Liste

$\begin{array}{lll}\begin{array}{l}\text { Sucre } \\ \text { farine }\end{array} & \begin{array}{l}\text { viande pour farce } \\ \text { steak haché 5\% }\end{array} & \begin{array}{l}\text { lessive } \\ \text { détachant }\end{array} \\ \begin{array}{l}\text { Huile d'olive } \\ \text { chapelure }\end{array} & \text { oignon } & \\ & & \end{array}$

(Orlea 2007, 131)

Une liste, bien que maigre, se trouve dans ce qui suit:

(15) Il ne sait pas ce qu'il fait dans cette chambre d'hôpital. Couché dans son lit, visage cireux, le malade égrène des noms de garages :

- Garage Delys, garage Gondin, garage Vireux, garage Petit, garage Mercier...

Delys, c'était en 42 ! Il les connaît ces noms de garage, ils figurent dans son propre dossier de retraite et ce pauvre type qui est en train de mourir, il le connait aussi, mais ce n'est pas pour autant qu'il n'en a pas marre de l'écouter [...]

-...garage Solen, garage Grimaux, garage Le Poitier...

(Orlea 2007, 130-131)

Dans « - Garage Delys, garage Gondin, garage Vireux, garage Petit, garage Mercier» il y a une unité typographique nette qui ne se laisse pas troubler par la mise en page sous forme de colonne, pendant que dans "- garage Solen, garage Grimaux, garage Le Poitier... », se trouve un fragment d'une unité typographique, marqué explicitement par des points de suspension au début comme à la fin.

Une opération d'ancrage référentiel, que l'on nomme opération de pré-thématisation, exige la présence d'un nom en tête, comme dans (3). Ce nom, nous l'avons affirmé plus haut, est une condensation de la description, un résumé, dans le sens que lui donnent Jean-Michel Adam et André Petitjean $(1989,115)$, encadré dans l'une des situations suivantes: «- la représentation descriptive 'joue' avec les connaissances du lecteur pour les confirmer ou les modifier ; - la représentation descriptive actualise une référence virtuelle sous la forme d'objets du discours ». 
(16) Bientôt Noël. Dans les vitrines, des fils dorés et de la fausse neige d'un blanc sale ont recouvert la laideur de la marchandise. De maigres sapins en plastique se dressent dans les recoins des étalages. Des queues houleuses ondulent sur les trottoirs qui longent les devantures des magasins d'alimentation. Les vieux jouent de leurs vieux genoux et de leurs coudes pointus pour évincer les jeunes, les jeunes jouent de leur mépris et de leurs muscles pour ne pas se laisser submerger par la violence désespérée des vieux. Les femmes, visages durs, regards de rapaces aux aguets, courent d'un bout à l'autre de la ville pour trouver de quoi bricoler le dîner du réveillon. [...]

Bientôt Noël. Le mince fil des lettres qui rattachait encore Léontine à

André s'était rompu [...] (Orlea 1986, 58-59)

La description de ce Noël aux couleurs de l'époque roumaine totalitaire argumente en modifiant une représentation universellement enracinée dans l'esprit, celle de la joie et du bonheur qui accompagnent une fête célébrée d'un bout à l'autre de la Terre. Tout en décrivant, on argumente. Même tout en listant, on argumente, comme dans (2) qui, dans son contexte réel spatio-temporel, est un texte-preuve de résistance politique.

L'affectation, opération inverse de l'ancrage, "produit des effets se sens type incertitude ou étrangeté » (Adam, Petitjean 1989, 115)

D’une manière très simple, l'affectation est définie comme une opération descriptive qui met en avant les propriétés et place le titre-thème à la fin. Si l'on prend en compte la compréhension de la séquence descriptive, il convient de « distinguer la référence virtuelle déclenchée par l'ancrage (attente d'une classe abstraite, d'un faisceau d'aspects) de la référence actuelle (la classe construite) produite par le déroulement même de la séquence descriptive » (Adam, Petijean 1989, 116)

«Mur, toit, porte, fenêtres, elle était là, devant nous, ni belle ni laide, et nous avons prononcé le mot, qu'un enfant de trois ans aurait: maison. » (Orlea 2007, 85). Cet exemple d'affectation place le thème-titre à la fin, mais présente également une particularité : la liste d'aspects n'enregistre que des éléments neutres, sans aucune particularisation, un schéma compositionnel de n'importe quelle maison; le manque de déterminant imposant le générique, idée renforcée par le négatif de la série : « ni belle ni laide ». La représentation descriptive qui s'ensuit est une confirmation (dans le sens d'Adam et Petijean) de la représentation déjà formée sur ce que c'est qu'une maison.

La reformulation. Comme l'affirme Jean-Michel Adam à juste titre, cette opération combine les deux procédures précédentes, « elle reprend en modifiant le thème titre initial (REFORMULATION)» $(2011,78)$

(17) Les escargots pestilentiels ont été chassés par un animal gigantesque et diffus, dont le corps a la forme d'une pluie inodore qui ne cesse plus de tomber sur la ville. Les gens se sont très vite rendu compte que la pluie n'était pas une pluie réelle, parce qu'il n'y avait ni gouttes ni 
accumulations d'eau. La pluie-animal a tout imbibé. Elle vit maintenant dans la matière même de la ville; dans la pierre des murs, dans le verre des fenêtres, dans l'asphalte des rues, dans le bois des arbres, dans l'eau des canaux, dans l'air que les gens respirent.

La pluie-animal se nourrit du contenu des choses. Elle vide, d'une façon extrêmement lente et imperceptible $[\ldots]$

La pluie-animal pénètre de plus en plus profond, de plus en plus loin. Il n'y a pas d'abri contre la pluie-animal, les gens ont tout essayé : les parapluies métalliques, les pèlerins cuirassés, les refuges souterrains blindés, la révolte et le silence. (Visniec 1996, 51-52)

Le passage de « animal gigantesque et diffus » à « la pluie-animal » garde un élément commun : animal signalant ainsi encore une fois la reformulation. Le parcours reformulatif ne sort pas des paramètres descriptifs : «dont le corps a la forme dune pluie inodore », " pas une pluie réelle », « la pluieanimal». Une fois introduite, la reformulation affecte toutes les propriétés: l'existence de la pluie, les sources de vie (se nourrit des contenus des choses), sa manière de se manifester.

(18) Car la pluie-animal vit aussi dans la chair des hommes, dans leur sang, dans leurs gestes et dans leurs rêves [...] Elle parle avec nous, comme si elle était une deuxième voix de notre être. Ses remarques sont encore assez primitives, comme, par exemple: "Monsieur, il ne faut pas peser à cela", ou "Oh, assez, vous allez trop loin" ou "C'est dangereux d'insister" ou carrément: "Laissez tomber, cela ne marchera pas non plus". (Visniec 1996, 52-53)

Le rôle attribué à la reformulation est celui d'ouvreur de paragraphes successifs, comme l'affirme Jean-Michel Adam (2011, 79) et le montre notre corpus : "La pluie-animal se nourrit [...] La pluie-animal pénètre $[\ldots]$ Car la pluie-animal $[\ldots] »$.

\section{Conclusions}

La complexité d'une structure descriptive repose sur l'énumération. Sa variété d'expression n'est pas si spectaculaire, mais elle est non plus une simple superposition d'images.

Dans le discours littéraire romanesque des écrivains roumains d'expression française, distinguer les régularités des descriptions et essayer une classification pour mettre un peu d'ordre dans leur manifestation nous a déterminé à considérer deux critères comme opératoires : l'organisation et la délimitation. Selon le premier une possible hiérarchie comprend la liste, l'inventaire et la description proprement dite, selon le deuxième, la classification est encore plus compliquée, mais nous permet de distinguer des énumérations clôturées et des énumérations ouvertes et encore d'autres, qui explicitent la clôture ou la fermeture.

Deux perspectives on été privilégiées dans leur étude: la perspective discursive basée sur l'étude de l'expression du point de vue et 
de l'aspect photographique et la perspective textuelle, focalisée sur les opérations qui assume la lisibilité de la séquence descriptive et organise sa linéarité.

\section{Textes de références}

Iulian, Rodica. Fin de chasse. Paris : L'Harmattan, 2001.

Lazar, Liliana. Terre des affranchis. Paris : Gaïa Editions, 2009.

Mailat, Maria. La Cuisse de Kafkea. Paris : Fayard, 2003.

Orlea, Oana. Un Sosie en cavale. Paris : Seuil, 1986.

Orlea, Oana. Rencontres sur le fil du rasoir. Paris : Gallimard, 2007.

Visniec, Matéi. Théatre décomposé ou L'Homme poubelle. Paris : L'Harmattan, 1996.

Tsepeneag, Dumitru. Roman de gare. Paris : P.O.L., 1985.

\section{Bibliographie}

Adam, Jean-Michel. Les Textes : types et prototypes. Paris : Armand Colin, 3 e édition, 2011.

Adam, Jean-Michel. La Description. Paris : PUF, 1993.

Adam, Jean-Michel, Petitjean, André. Le Texte descriptif. Poétique historique et linguistique textuelle. Paris : Nathan, 1989.

Adam, Jean-Michel, Revaz, Françoise, «Aspects de la structuration du texte descriptif: les marqueurs d'énumération et de reformulation». Langue française, no 81, 1989: 59-98. [Texte en ligne]. URL: http://www.persee.fr/web/revues/home/prescript/issue/lfr_00238368_1989_num_81_1 (Consulté le 1 août 2013).

Charaudeau, Patrick, Maingueneau, Dominique (éds.). Dictionnaire d'analyse $d u$ discours. Paris : Seuil, 2002.

Hamon, Philipe. "La Description de l'indescriptible». Compte rendu de la communication au Groupe Hugo du 19 mai 1990. [Texte en ligne]. URL: http://groupugo.div.jussieu.fr/groupugo/90-05-19hamon.htm (Consulté le 1er août 2013).

Jeandillou, Jean-François. L'Analyse textuelle. Paris : Armand Colin, 1997.

Maingueneau, Dominique. Analyse du discours. Introduction aux lectures de l'archive. Paris : Hachette, 1991.

Paveau, Marie-Anne, Rosier, Laurence. « Grammaire de la liste » in Evrard, Ivan, Pierrard, Michel, Rosier Laurence, Raemdonck, Dan van, Le Sens en marge. Représentations linguistiques et observables discursifs. Paris : l'Harmattan, 2009 : 113135. 